\title{
Opponent chromatic response functions for an average observer
}

\author{
JOHN S. WERNER and B. R. WOOTEN \\ Laboratory of Psychology, Brown University, Providence, Rhode Island 02912
}

\begin{abstract}
Average opponent chromatic response functions are presented for use in quantitative models of color perception. Theoretical transformations of photopigment input are related to opponent channel activity. Opponent chromatic activity is, in turn, used to describe a theoretical huenaming curve for an average observer.
\end{abstract}

In quantitative theoretical formulations, it is often useful to assume psychophysical functions for an average, or standard, observer. In vision, one may find such data for a number of phenomena: scotopic spectral sensitivity, photopic spectral sensitivity for normal and dichromatic observers, color mixture wavelength discrimination, preretinal optic media, and receptor spectral sensitivity. Most of these data describe early stages in visual processing. Yet, it is now clear from psychophysical and electrophysiological studies that an opponent transformation, such as that postulated by Hering $(1920,1964)$ occurs subsequent to quantal absorption. This opponent transformation is directly related to hue perception. In order to evaluate quantitative models of hue processing, one must assume, or measure directly a set of opponent chromatic response functions. In this paper, we present opponent response functions based upon an average of all data available from the literature.

\section{THE OPPONENT-RESPONSE MODEL}

Hering postulated that hue is coded by the activity in neural opponent chromatic channels, a redgreen channel and a yellow-blue channel. Each of these channels has two antagonistic modes of response. For example, when the yellow-blue channel is activated to signal yellowness, it cannot simultaneously signal blueness. The sensation of yellowness cancels the sensation of blueness, as is evidenced by the achromatic sensation resulting from the addition of yellow and blue lights. Similarly, the sensations of redness and greenness are opponently organized.

Jameson and Hurvich (1955) capitalized on this antagonism when they measured the response functions of the opponent chromatic channels. To measure the strength of blueness at a particular wavelength, they determined the radiance of a superposed yellow light required to cancel the sensation of blue-

J. S. Werner's present address is: Department of Psychology, University of Colorado, Boulder, Colorado 80309. ness. The assumption was that the strength of blue activity at a particular wavelength is proportionate to the radiance of yellow required for cancellation. In a similar manner, opponent hue cancellation was used to measure the strength of the red-green channel. In addition to providing the first quantitative measurements of the opponent chromatic channels, Jameson and Hurvich (1955, 1956; Hurvich \& Jameson, 1955, 1956) also proposed specific quantitative relations for normal and color deficient observers between opponent mechanisms and saturation, brightness, wavelength discrimination, perceived hue, and adaptation effects.

The cancellation method of Jameson and Hurvich was subsequently employed by Romeskie (1978) and by Werner and Wooten (1979) to measure opponent mechanisms in other normal trichromatic observers. The average response functions from these three studies (seven observers) are presented in Table 1 and Figure 1. ${ }^{1}$ The average wavelength locus for the unique hues, which were measured independently for the same seven observers, lies where one of the average opponent response functions is in equilibrium. These points are: unique blue, $471 \mathrm{~nm}$; unique green, $507 \mathrm{~nm}$; and unique yellow, $577 \mathrm{~nm}$.

The specific experimental methods were similar among the three studies, with two exceptions: First, Jameson and Hurvich presented their monochromatic test flashes with a chromatically neutral white surround field, while the other two studies used an isolated stimulus (i.e., no background). The effect of this variable has not been investigated. Second, Werner and Wooten used 1-sec stimulus presentations, while the other two studies used flash durations of several seconds. Werner and Wooten showed that the stimulus exposure duration used in these studies is not a significant parameter in contributing to observer differences (over the range of 1 to $7 \mathrm{sec}$ ), at least for the yellow-blue channel. Despite observer differences within and between studies, the general form of the opponent cancellation functions is similar for 

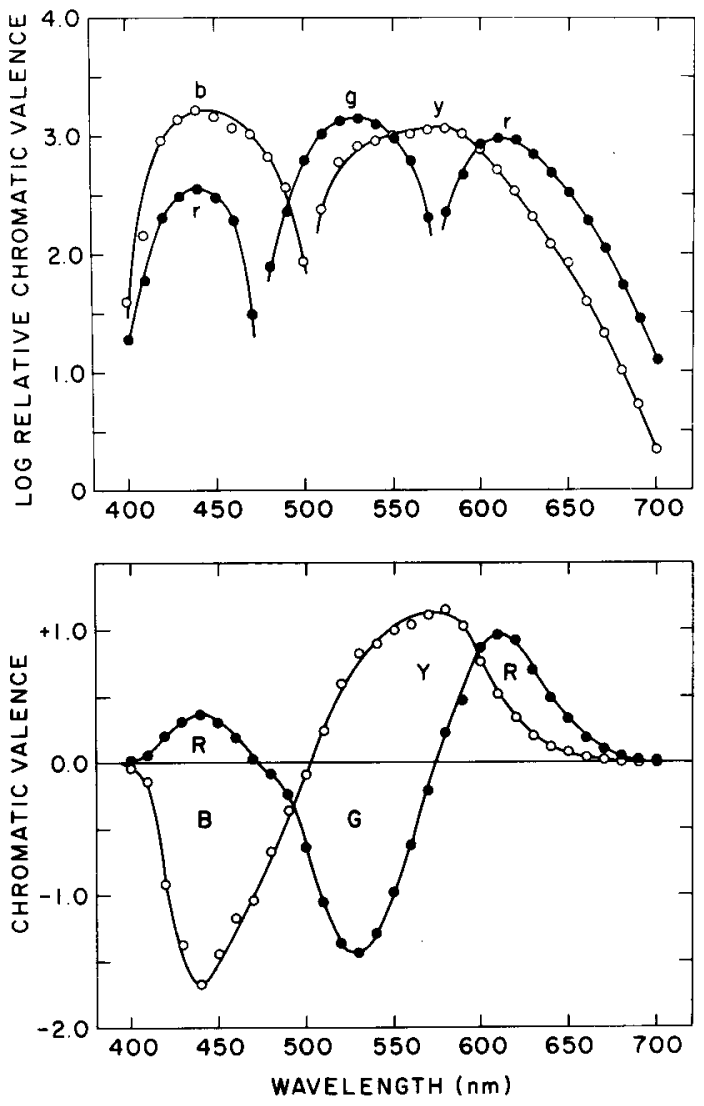

Figure 1. Opponent chromatic cancellation functions for the average observer plotted for an equal-energy spectrum. The top panel presents these functions on logarithmic ordinates; the bottom panel shows the data on arithmetic ordinates. The redgreen system is plotted with closed circles and the yellow-blue system is shown by open circles.

all observers. The average response function may, therefore, be useful for quantitative modeling.

\section{THEORETICAL PHOTOPIGMENT RELATIONS}

Jameson and Hurvich (1968) have proposed that the red-green and yellow-blue opponent channels are linearly related to quantal absorption of the three cone photopigments. The data of several studies (Larimer, Krantz, \& Cicerone, 1974; Romeskie, 1978; Werner \& Wooten, 1979) indicate that the linear model adequately relates the photopigments to the red-green system. Romeskie was also able to fit yellow-blue chromatic response functions with a linear combination of the photopigments; however, the data of Larimer, Krantz, and Cicerone (1975) and of Werner and Wooten suggest that a nonlinear interaction between the mid- and long-wave cones is required to fit the yellow lobe of the yellow-blue channel.

To evaluate the theoretical photopigment models in relation to the average opponent response functions, a computer program was used to generate weighting coefficients for the short-, middle-, and long-wave cone photopigments (denoted in the equations by $\alpha, \beta$, and $\gamma$, respectively) to predict the opponent response functions using a least-squares criterion. These photopigments were based on an iodopsin nomogram (Wald, Brown, \& Smith, 1955) with $\lambda_{\max }$ at 435 , 530 , and $562 \mathrm{~nm}$. Wyszecki and Stiles' (1967) average lens and macular pigment absorption estimates were used to provide receptor action spectra relative to an equal-energy spectrum at the cornea. The computations were carried out only for points on which the average was based on seven observers. This analysis yielded the following linear functions for the red-green (Equation 1) and yellow-blue systems (Equation 2):

$$
\begin{aligned}
& (\mathrm{r}-\mathrm{g})_{\lambda}=0.45 \alpha_{\lambda}-2.79 \beta_{\lambda}+1.89 \gamma_{\lambda} \\
& (\mathrm{y}-\mathrm{b})_{\lambda}=-1.72 \alpha_{\lambda}+0.22 \beta_{\lambda}+0.85 \gamma_{\lambda}
\end{aligned}
$$

As shown in Figure 2, the fit for the r-g system is somewhat better than that for the $y-b$ system. The error (residuals divided by number of data points) for the r-g linear fit was $\mathbf{. 0 0 3}$, and for the y-b system it was .018. The error for the y-b system was

Table 1

\begin{tabular}{|c|c|c|}
\hline Wavelength & r-g Valence & $y-b$ Valence \\
\hline 400 & $(+.0192)$ & $(-.0397)$ \\
\hline 410 & $(+.0600)$ & $(-.1442)$ \\
\hline 420 & +.2045 & -.9173 \\
\hline 430 & +.3117 & -1.3733 \\
\hline 440 & +.3659 & -1.6684 \\
\hline 450 & +.3050 & -1.4414 \\
\hline 460 & +.1958 & -1.1773 \\
\hline 470 & $(+.0314)$ & -1.0382 \\
\hline 480 & $(-.0789)$ & -.6719 \\
\hline 490 & -.2333 & -.3659 \\
\hline 500 & -.6319 & $(-.0871)$ \\
\hline 510 & -1.0514 & $(+.2426)$ \\
\hline 520 & -1.3590 & $(+.5931)$ \\
\hline 530 & -1.4295 & $(+.8202)$ \\
\hline 540 & -1.2827 & +.8952 \\
\hline 550 & -.9715 & +1.0000 \\
\hline 560 & -.6189 & +1.0391 \\
\hline 570 & $(-.2068)$ & +1.1095 \\
\hline 580 & $(+.2285)$ & +1.1515 \\
\hline 590 & +.4723 & +1.0317 \\
\hline 600 & +.8663 & +.7640 \\
\hline 610 & $\begin{array}{r}+.9689 \\
\end{array}$ & +.5141 \\
\hline 620 & $\begin{array}{r}+.9269 \\
\end{array}$ & +.3380 \\
\hline 630 & +.7025 & +.2048 \\
\hline 640 & +.4902 & +.1201 \\
\hline 650 & +.3341 & +.0838 \\
\hline 660 & $\begin{array}{r}.1921 \\
+\quad .192\end{array}$ & +.0393 \\
\hline 670 & $\begin{array}{r}.1119 \\
+\quad .11\end{array}$ & +.0210 \\
\hline 680 & +.0547 & +.0102 \\
\hline 690 & +.0287 & +.0053 \\
\hline 700 & +.0128 & +.0022 \\
\hline
\end{tabular}

Average Opponent Chromatic Valence 

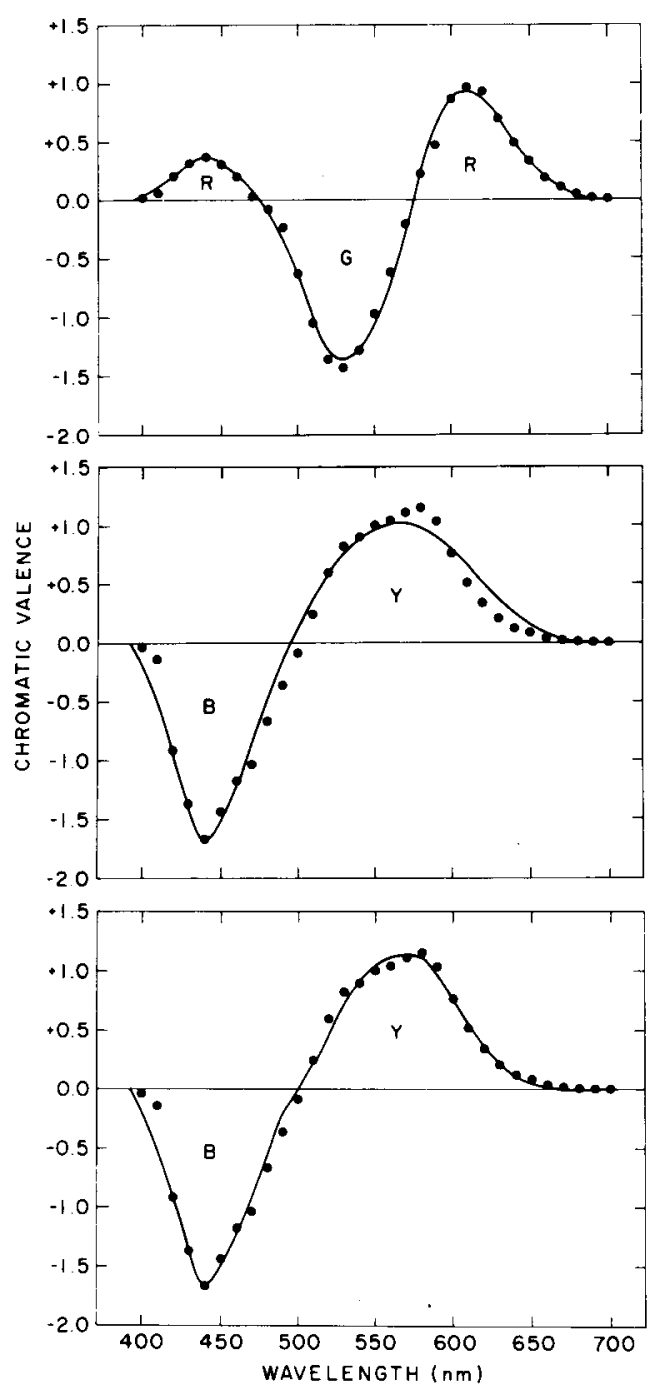

Figure 2. Opponent chromatic cancellation functions for the average observer plotted for an equal-energy spectrum. Top panel: Smooth curve is the best fitting linear combination of the photopigments to the red-green channel (Equation 1). Middle panel: Smooth curve is the best fitting linear combination of the photopigments to the yellow-blue channel (Equation 2). Bottom panel: Smooth curve is the best fitting nonlinear combination of the photopigments to the yellow-blue channel (Equation 3).

reduced to .010 by the nonlinear function (see Werner $\&$ Wooten for a discussion of this model):

$$
(y-b)_{\lambda}=-1.65 \alpha_{\lambda}+\left|0.21 \beta_{\lambda}+0.94 \gamma_{\lambda}\right|^{1.65} \text {. }
$$

A qualitative comparison of the fits with the linear and nonlinear models to the y-b system can be made by comparing the middle and bottom panels of Figure 2.

\section{THEORETICAL HUE RELATIONS}

Hurvich and Jameson (1955) have proposed a model relating opponent chromatic activity to per- ceived hue. According to their model, perceived redness or greenness, or a hue coefficient for redness or greenness, is determined by the ratio of red-green opponent activity to the sum of all opponent chromatic activity. A similar relation was proposed for perceived yellowness or blueness. Their model is formally described by the equations:

$$
h_{(r, g)_{\lambda}}=\frac{(|r-g|)_{\lambda}}{(|r-g|+|y-b|)_{\lambda}}
$$

and

$$
h_{(y, b)_{\lambda}}=\frac{(|y-b|)_{\lambda}}{(|r-g|+|y-b|)_{\lambda}}
$$

In this model, the sum of the hue coefficients, $h_{(r-g)}$ and $h_{(y-b)_{\lambda}}$, is equal to unity.

Werner and Wooten used this model to relate individual differences in opponent activity to individual differences in perceived hue. Perceived hue was measured by a hue-naming task in which observers described the percentage of red, green, blue, or yellow in monochromatic, equal-luminance lights. Opponent cancellation predicted hue naming to within approximately $10 \%$ for each observer. Since opponent cancellation accurately predicts perceived hue, Equation 4 and the average opponent response functions can be used to derive a theoretical hue-naming function for the average observer. This function is shown in Figure 3. Empirically determined hue naming data from Werner and Wooten's three observers agree well with this theoretical function. The mean difference between these two sets of points is $9.6 \%$. The theoretical hue-naming function for the average observer was also compared with a hue-naming

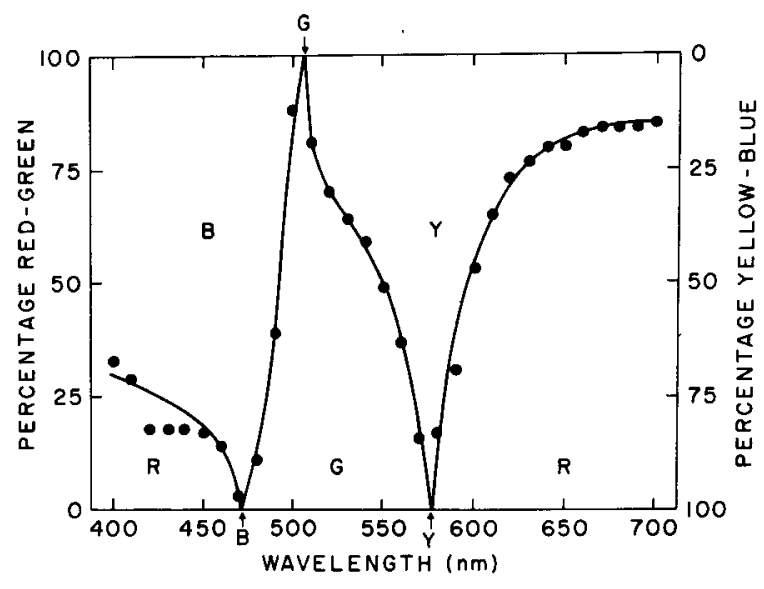

Figure 3. Theoretical hue-naming data derived by Equation 4 and the opponent response functions of the average observer. Redgreen is plotted from $0 \%$ to $100 \%$ according to the left vertical axis and yellow-blue is plotted from $100 \%$ to $0 \%$ according to the right vertical axis. The arrows indicate the average unique hue loci. The smooth curve was drawn through the data points by eye. 
function derived by Hurvich and Jameson (1955) from Judd's (1951) transformation of the $2^{\circ}$ C.I.E. ${ }^{2}$ stimulus values. The average difference between these two theoretical functions was $7.6 \%$. Thus, theoretical predictions of perceived hue on the basis of the average opponent response functions agree fairly well with other empirical and theoretical determinations.

\section{REFERENCES}

HeRING, E. [Outlines of a theory of the light sense] (L. M. Hurvich \& D. Jameson, trans.). Cambridge, Mass: Harvard University Press, 1964. (Originally published, 1920.)

HuRvich, L. M., \& Jameson, D. Some quantitative aspects of an opponent-colors theory. II. Brightness, saturation, and hue in normal and dichromatic vision. Journal of the Optical Society of America, 1955, 45, 602-616.

HuRvich, L. M., \& J JMeson, D. Some quantitative aspects of an opponent-colors theory. IV. A psychological color specification system. Journal of the Optical Society of America, 1956, 46, 416-421.

Jameson, D., \& Hurvich, L. M. Some quantitative aspects of an opponent-colors theory. I. Chromatic responses and spectral saturation. Journal of the Optical Society of America, 1955, 45, 546-552.

Jameson, D., \& Hurvich, L. M. Some quantitative aspects of an opponent-colors theory. III. Changes in brightness, saturation, and hue with chromatic adaptation. Journal of the Optical Society of America, 1956, 46, 405-415.

Jameson, D., \& Hurvich, L. M. Opponent-response functions related to measured cone photopigments. Journal of the Optical Society of America, 1968, 58, 429-430.

JuDD, D. B. Basic correlates of the visual stimulus. In S. S. Stevens (Ed.), Handbook of experimental psychology. New York: Wiley, 1951.

Larimer. J., Krantz, D. H., \& Cicerone, C. M. Opponent process additivity-I. Red/green equilibria. Vision Research, 1974, 14. $1127-1140$.
Larimer, J., Krantz, D. H., \& Cicerone, C. M. Opponent process additivity-II. Yellow/blue equilibria and nonlinear models. Vision Research, 1975, 15, 723-731.

Romeskie, M. Chromatic opponent-response functions of anomalous trichromats. Vision Research, 1978, 18, 1521-1532.

Wald, G., Brown, P. K., \& SMITh, P. H. Iodopsin. Journal of General Physiology, 1955, 38, 623-681.

Werner, J. S., \& Wooten, B. R. Opponent chromatic mechanisms: Relation to photopigments and hue naming. Journal of the Optical Society of America, 1979, 69, 422-434.

Wyszecki, G., \& Stiles, W. S. Color science: Concepts and methods, quantitative data and formulas. New York: Wiley, 1967.

\section{NOTES}

1. Points for which the average is based on less than seven observers are specified in Table 1 by parentheses. The minimum number of observers at these points is three. At midwaves, the $\mathrm{N}$ was reduced because of individual differences in the locus of the crossover points within channels (e.g., between red and green, and between yellow and blue). For these points, the average was computed with all available data by assigning opposite signs for opposite members of an opponent pair. It should also be noted that in order to present the data in 10-nm steps, it was necessary to interpolate between points at long waves for Romeskie's two observers.

2. Judd's theoretical opponent chromatic response functions are based upon the following linear transformation of the C.I.E. tristimulus values:

$$
(\mathrm{r}-\mathrm{g})_{\lambda}=1.0 \overline{\mathrm{x}}_{\lambda}-1.0 \overline{\mathrm{y}}_{\lambda}
$$

and

$$
(y-b)_{\lambda}=0.4 \overline{y_{\lambda}}-0.4 \overline{z_{\lambda}}
$$

(Received for publication December 5, 1978; revision accepted January $9,1979$. 